\title{
A Nonribosomal Peptide Synthase Containing a Stand-Alone Condensation Domain Is Essential for Phytotoxin Zeamine Biosynthesis
}

\author{
Yingying Cheng, ${ }^{1}$ Xiaoling Liu, ${ }^{2}$ Shuwen An, ${ }^{2}$ Changqing Chang, ${ }^{2}$ Yuanqiang Zou, Luhao Huang, ${ }^{1}$ \\ Jin Zhong, ${ }^{1}$ Qiongguang Liu, ${ }^{1}$ Zide Jiang, ${ }^{1}$ Jianuan Zhou, ${ }^{1}$ and Lian-Hui Zhang ${ }^{1,2}$ \\ ${ }^{1}$ Guangdong Province Key Laboratory of Microbial Signals and Disease Control, South China Agricultural University, Guangzhou \\ 510642, People's Republic of China; ${ }^{2}$ Institute of Molecular and Cell Biology, 61 Biopolis Drive, Singapore 138673
}

Submitted 9 April 2013. Accepted 4 July 2013.

Dickeya zeae is the causal agent of rice foot rot and maize stalk rot diseases, which could cause severe economic losses. The pathogen is known to produce two phytotoxins known as zeamine and zeamine II which are also potent antibiotics against both gram-positive and gram-negative bacteria pathogens. Zeamine II is a long-chain aminated polyketide and zeamine shares the same polyketide structure as zeamine II, with an extra valine derivative moiety conjugated to the primary amino group of zeamine II. In this study, we have identified a gene designated as $z m s K$ encoding a putative nonribosomal peptide synthase (NRPS) by screening of the transposon mutants defective in zeamine production. Different from most known NRPS enzymes, which are commonly multidomain proteins, ZmsK contains only a condensation domain. High-performance liquid chromatography and mass spectrometry analyses showed that the ZmsK deletion mutant produced only zeamine II but not zeamine, suggesting that $\mathrm{ZmsK}$ catalyzes the amide bond formation by using zeamine II as a substrate to generate zeamine. We also present evidence that a partially conserved catalytic motif within the condensation domain is critical for zeamine production. Furthermore, we show that deletion of $z m s K$ substantially decreased the total antimicrobial activity and virulence of D. zeae. Our findings provide a new insight into the biosynthesis pathway of zeamines and the virulence mechanisms of the bacterial pathogen $D$. zeae.

Dickeya zeae, formerly known as Erwinia chrysanthemi pv. zeae (Samson et al. 2005), is the causal agent of rice foot rot and maize stalk rot diseases. The pathogen has been found in various parts of the world, including the Middle East, Europe, Africa, America, and Asia, and become a serious concern of the agricultural industry in the rice- and corn-planting countries and regions (Goto 1979; Liu and Wang 2004; Nassar et al. 1994; Stead et al. 2010). Notably, different to the other species in Dickeya family, which infect only dicotyledons, D. zeae infects not only monocotyledons but also dicotyledons (Goto 1979; Hussain et al. 2008; Sinha and Prasad 1977). Investi-

Corresponding authors: L.-H. Zhang; E-mail: lianhui@imcb.a-star.edu.sg; and J. Zhou; E-mail: Jianuanzhou@scau.edu.cn

* The $\boldsymbol{e}$-Xtra logo stands for "electronic extra" and indicates that two supplementary figures and one supplementary table are published online.

(C) 2013 The American Phytopathological Society gation of its host specificity and pathogenic mechanisms would be essential for the control and prevention of the rice foot rot and maize stalk rot diseases.

Recently, we have identified two polyketide phytotoxins (i.e., zeamine and zeamine II) which are also potent antibiotics against a range of gram-positive and gram-negative bacterial pathogens (Wu et al. 2010; Zhou et al. 2011). The zeamineminus mutants are unable to inhibit rice seed germination and show substantially decreased virulence on potato and Chinese cabbage (Zhou et al. 2011), suggesting that zeamines are the major virulence factors of $D$. zeae. The two zeamines are structurally similar, with zeamine II being a long-chain aminated polyketide containing a secondary alcohol, one primary, and four secondary amines, and zeamine sharing the same chemical structure as zeamine II, with an 6-amino-3,5-dihydroxy-7methyloctanoate (octanoate) extension. An isotope-labeled substrate feeding test showed that the octanoate moiety of zeamine is a short polyketide with valine as an initiating unit (Wu et al. 2010). The findings suggest that zeamine II may be an intermediate in biosynthesis of zeamine. Consistent with this notion, mutation of $z m s A$, which encodes a novel polyketide synthase, abolishes the production of both zeamine and zeamine II (Zhou et al. 2011). These structural features of zeamines also suggest that there may be a nonribosomal peptide synthase (NRPS) that catalyzes the condensation of the aminated polyketide moiety with the octanoate group in the biosynthesis of zeamine.

To investigate the biosynthetic pathway of zeamines, we generated a transposon mutant library using $D$. zeae strain $\mathrm{EC} 1$ as the parental strain, and screened for the mutants with altered production of zeamines. In this study, we identified and characterized a new NRPS gene designated as $z m s K$ which encodes a peptide containing only a condensation domain. Null mutation of $z m s K$ resulted in decreased antibiotics activity and partially attenuated virulence on rice and tobacco. High-performance liquid chromatography (HPLC) and mass spectrometry (MS) analyses showed that the zmsK deletion mutant produced only zeamine II but not zeamine, indicating that ZsmK is the key enzyme for the biosynthesis of zeamine.

\section{RESULTS}

Identification of a $D$. zeae mutant showing partially decreased antimicrobial activity.

To understand the biosynthetic mechanisms of zeamines, we used the Tn5 random insertion mutagenesis approach to generate a mutant library for screening of the genes associated with 
the production of zeamines. By using Escherichia coli as an indicator strain, we identified a range of transposon mutants with altered antimicrobial activity. Among them, we found that the mutant EM104 displayed a compromised antimicrobial activity compared with the D. zeae wild-type strain EC1 (Fig. 1A). This is distinct from the mutant phenotype of $z m s A$ (National Center for Biotechnology Information [NCBI] accession number KF214760), which is the first characterized gene involved in zeamine biosynthesis. We showed recently that null mutation of zmsA led to a complete loss of zeamines production and the antimicrobial activity (Zhou et al. 2011). The findings suggest that the mutated gene in EM104 might play a different role in the biosynthesis of zeamines compared with $z m s A$. Thus, EM104 was selected for further characterization. The flank sequences of Tn 5 insertion were amplified by hithermal asymmetrical interlaced polymerase chain reaction (PCR) (Liu and Chen 2007). Analysis of the sequence data revealed that the transposon was inserted at the $906 \mathrm{bp}$ of an open reading frame (ORF) consisting of 1,494 bp nucleotides, which encodes a peptide of 497 amino acids (aa). The GC content of this gene is $49.06 \%$, which is comparable with the $48.23 \%$ GC percentage of $z m s A$. A ribosome binding site motif (GGAG) was found at $12 \mathrm{bp}$ upstream of the start codon ATG of the ORF, and this start codon is overlapped with the termination codon of the upstream gene (Supplementary Fig. S1). As there are at least nine ORF between $z m s A$ and this ORF according to our unpublished partial genome sequence data, we designated this ORF as $z m s K$ (NCBI accession number KF214759) for the convenience of discussion.

To verify the role of $z m s K$ in zeamine biosynthesis, we generated an in-frame deletion mutant by deleting an internal fragment corresponding to the 410th to the 1,117th bp of the ORF. A bioassay against $E$. coli $\mathrm{DH} 5 \alpha$ showed that $z m s K$ deletion resulted in partial reduction of the total antimicrobial activity of zeamines (Fig. 1B). A semiquantitative analysis showed that deletion of the $z m s K$ gene caused approximately $57 \%$ reduction in antimicrobial activity, whereas in trans expression of the wild-type $z m s K$ gene restored the activity of zeamines to a level approximately $34 \%$ higher than that of the wild-type strain EC1 (Fig. 1A).

\section{Protein in silico analysis.}

Domain structure analysis showed that $\mathrm{ZmsK}$ contains only a condensation domain from the 13th to the 308th aa (Fig. 2), suggesting that it is likely a member of NRPS family. A Blast search showed that the closest homologues of ZmsK are a condensation domain-containing protein Zmn19 from Serratia plymuthica AS12 (NCBI accession number YP_004502676). Zmn 19 contains 512 aa and shares approximately $48 \%$ identity in amino acids with $\mathrm{ZmsK}$ with $96 \%$ coverage but its enzymatic role has not yet been characterized. The other homologues are less similar to ZsmK, with amino acid identity of 25 to $31 \%$ and less query coverage of 61 to $73 \%$. Among them, the enzyme products of several proteins have been characterized, including the cryptophycin synthase CrpD (NCBI accession number ABM21572.1) from Nostoc sp. strain ATCC 53789, the n-(5-amino-5-carboxypentanoyl)-I-cysteinyl-d-valine synthase (NCBI accession number YP_003953768.1) from Stigmatella aurantiaca DW4/3-1, the tyrocidine synthase from Corallococcus coralloides DSM 2259 (NCBI accession number YP_005368578), the syringopeptin synthetase C from Pseudomonas syringae pv. syringae (NCBI accession number AAO72425.1), and the bacitracin synthetase 1 from $S$. aurantiaca DW4/3-1 (NCBI accession number YP_003950775.1). Domain analysis showed that all these characterized homologues contain other domains in addition to the condensation domain (Fig. 2A). Considering that the octanoate moiety in zeamine is derived from valine according to ${ }^{13} \mathrm{C}$-labeled valine feeding analysis (Wu et al. 2010), it is interesting to note that most products of these homologues contain a valine residue (Fig. 2B).

\section{Null mutation of ZmsK blocks zeamine production.}

Given that NRPS with a condensation domain is associated with catalysis of amide bond formation (Stachelhaus et al. 1998), we speculated that ZmsK may be responsible for the condensation reaction by using zeamine II and octanoate as substrates. To test the possibility, we compared the zeamine production profiles of the $z m s K$ mutant and its parental wildtype strain EC1. As previously reported (Zhou et al. 2011), HPLC analysis showed that strain EC1 produced two major peaks showing strong antimicrobial activity, with elution time at 28.7 and $31.4 \mathrm{~min}$, respectively (Fig. 3A). In contrast, the $z s m K$ mutant produced only one peak at 28.7 min, with strong antimicrobial activity (Fig. 3B). MS analysis showed that the MS signals (electrospray ionization) for the peak at $31.4 \mathrm{~min}$ were $841.8[\mathrm{M}+\mathrm{H}] / \mathrm{e}, 421.6[\mathrm{M}+2 \mathrm{H}] / 2 \mathrm{e}$, and $281.6[\mathrm{M}+3 \mathrm{H}] / 3 \mathrm{e}$ (Fig. 4A), which is agreeable with the calculated positive ion signals of zeamine, whereas the MS signals of the peak at 28.7 $\min$ were $654.7[\mathrm{M}+\mathrm{H}] / \mathrm{e}, 327.8[\mathrm{M}+2 \mathrm{H}] / 2 \mathrm{e}$, and 218.9 $[\mathrm{M}+3 \mathrm{H}] / 3 \mathrm{e}$ (Fig. 4B), which are typical for zeamine II according to our previous results (Zhou et al. 2011). Taken together, these results indicate that deletion of the $z m s \mathrm{~K}$ gene completely abolished zeamine biosynthesis but the mutant could still produce zeamine II.

\section{The partially conserved "PHxxxDG" motif is essential for the enzyme activity of ZmsK.}

Typically, NRPS is a multi-module enzyme which normally contains adenylation, peptidyl-carrier-protein, and condensation domain at least; some NRPS may contain epimerization, methylation, and other editing domains for special chemical reactions (Sieber and Marahiel 2005). The condensation domain catalyzes the amide bond formation between the thioester groups of the growing peptide chain from the previous module with the amino group of the current module. The condensation
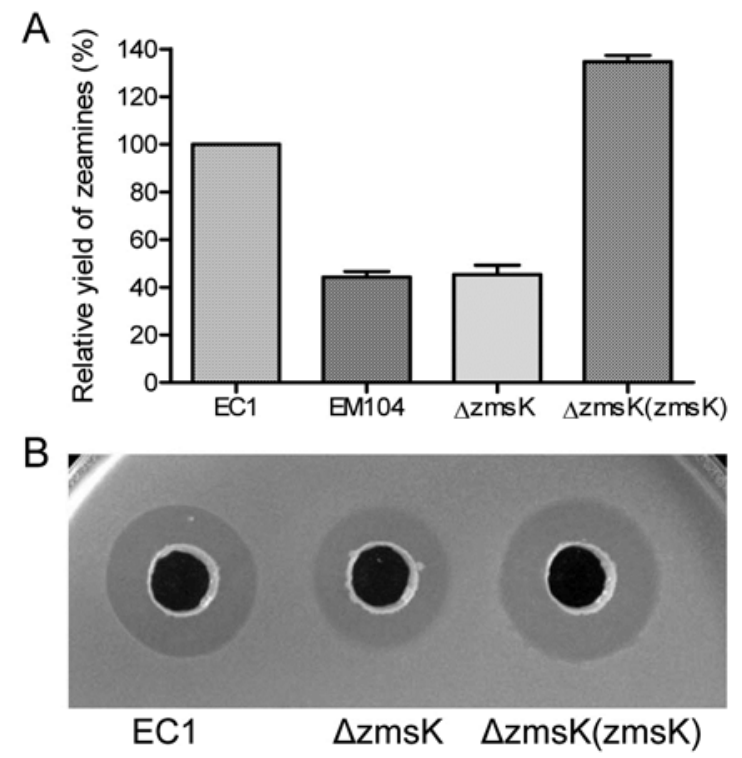

Fig. 1. Mutation of zsmK resulted in reduced antimicrobial activity. A, Quantitative comparison of zeamine production in Dickeya zeae wild-type strain EC1 and derivatives. The experiment was repeated three times with triplicates each time. Data shown are means of three repeats and error bars indicate standard deviations. B, Representative bioassay plate. The photograph was taken $24 \mathrm{~h}$ after inoculation. 
domain normally contains a conserved "HHxxxDG" motif that is the active site for catalysis of peptide bond formation (Stachelhaus et al. 1998). Sequence alignment of the condensation domain of $\mathrm{ZmsK}$ with other characterized condensation domains showed that $\mathrm{ZmsK}$ contains a partially conserved motif " ${ }^{145} \mathrm{PHxxxDG}^{151 "}$ at the center of the condensation domain
(13 to 308 aa) (Fig. 5A). To determine the role of this putative catalytic motif, we conducted a site-directed mutagenesis by substituting the "P", " $H$ ", "D", and "G" residues with alanine and used these mutated $z m s K$ derivatives for complementation analysis. Bioassay results support the critical role of this motif in zeamine biosynthesis. Compared with the complemented

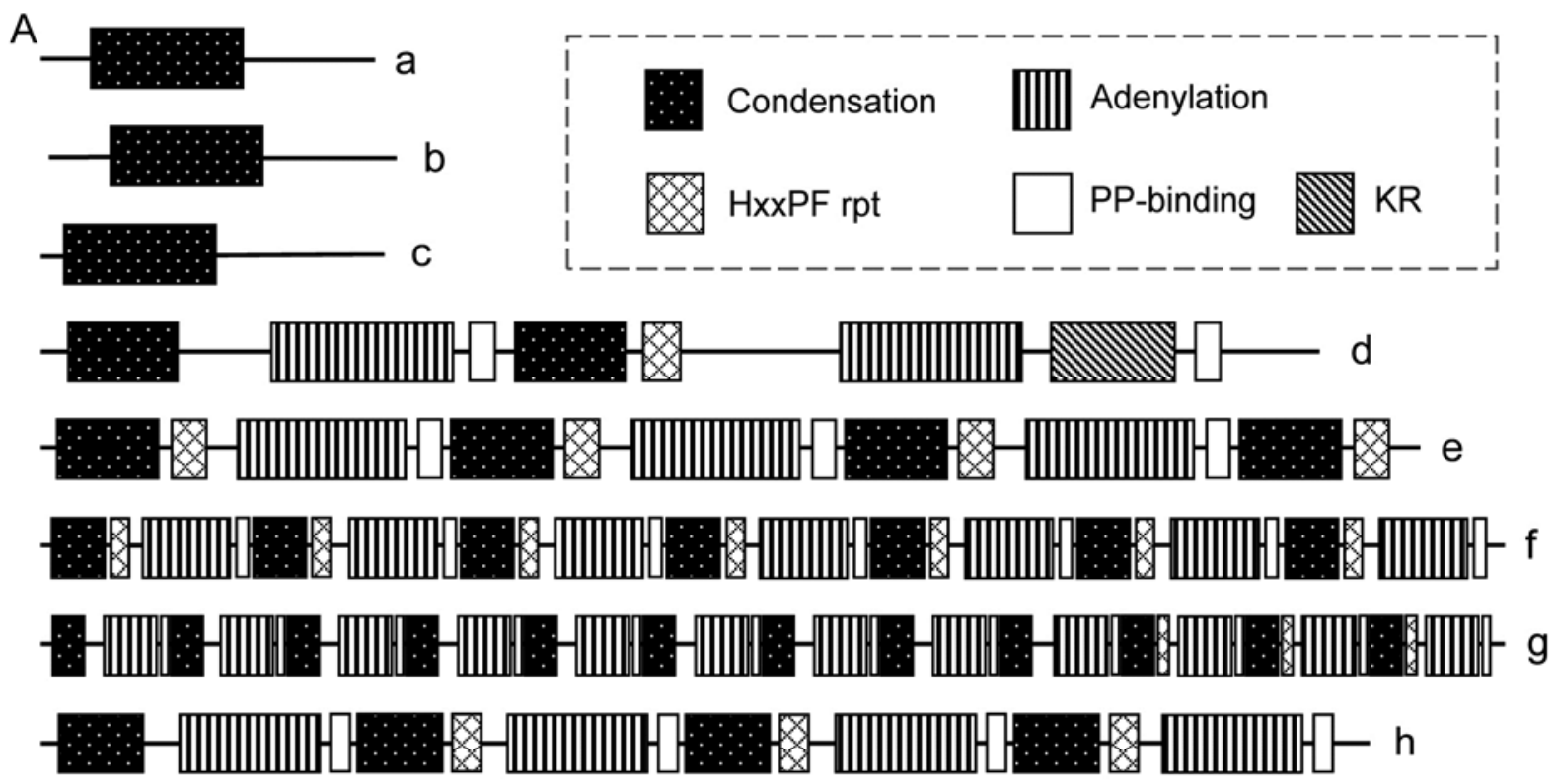

B

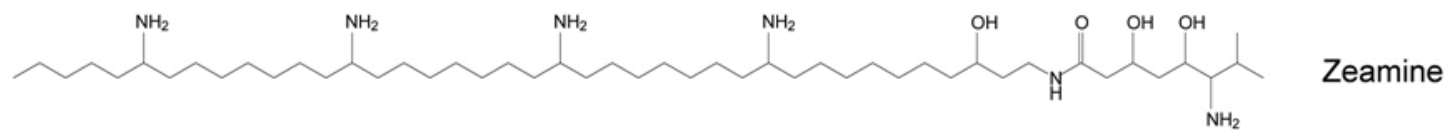<smiles>CCCCCC(N)CCCCCCCC(N)CCCCCCCCC(N)CCCCCCCC(N)CCCCCCCC(O)CCN</smiles>

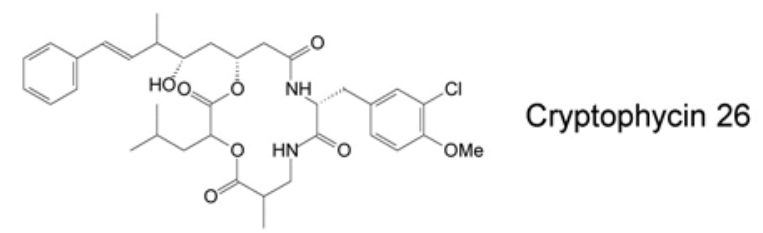

No

Phe-Pro-Phe-Phe-Asn-Gn-Tyr-Val-Om-Leu Tyrocidine
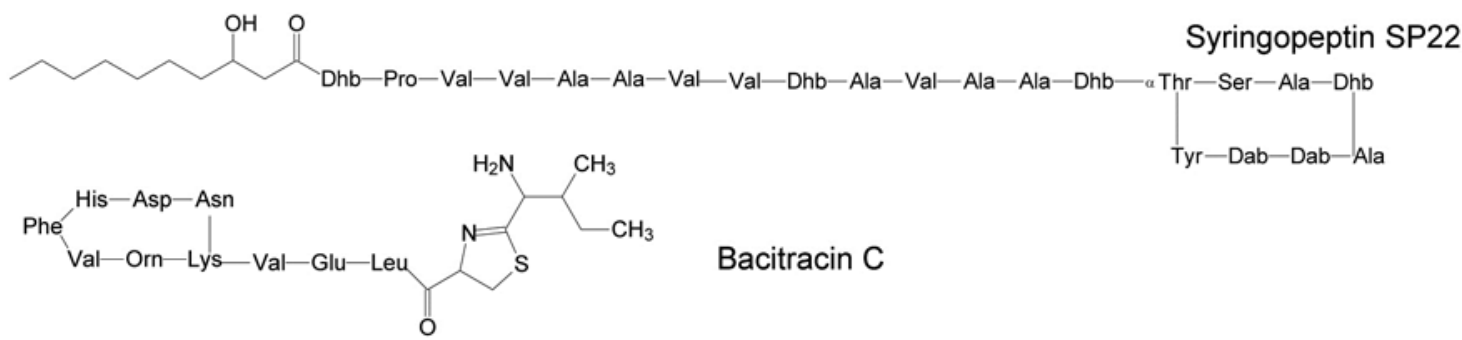

Fig. 2. Domain structures of ZmsK and homologues and related chemicals. A, Domain structures of ZmsK and homologues predicted by using SMART program, including row a, ZmsK; row b, Zmn19 from Serratia plymuthica AS12 (National Center for Biotechnology Information [NCBI] number YP 004502676); row c, the VibH from Vibrio cholera (NCBI number AAD48879.1); row d, the cryptophycin synthase CrpD from Nostoc sp. strain ATCC 53789 (NCBI number ABM21572.1); row e, the n-(5-amino-carboxypentanoyl)-1-cyteinyl-d-valine synthase (NcvS) from Stigmatella aurantica DW4/3-1 (NCBI number YP_003953768.1); row f, the tyrocidine synthase (TyrS) from Corallococcus coralloides DSM 2259 (NCBI number YP_005368578.1); row g, the syringopeptin synthase C (SysC) from Pseudomonas syringae pv. syringae (NCBI number AAO72425.1); and row h, the bacitracin synthetase 1 (BacS) from S. aurantica DW4/3-1 (NCBI number YP_003950775.1). B, Chemical structures of zeamine and zeamine II, and various chemical molecules produced by ZmsK homologues. 
strain $\Delta z m s K(z m s K)$ expressing the wild-type $z m s K$ gene, the P145A, H146A, D150A, and G151A substitution reduced the zeamine production by approximately $15,22,43$, and $11 \%$, respectively (Fig. 5B). When all four residues were substituted by alanine at the same time, the total activity of zeamines was decreased by approximately $60 \%$ compared with the strain expressing the wild-type $z m s K$ gene, which is lower than any single mutant (Fig. 5B).

\section{Deletion of $z m s K$ attenuates bacterial virulence.}

Our previous study showed that mutation of the polyketide synthase gene $z m s A$ in strain EC1 abolished the production of zeamines and the virulence against rice seed germination (Zhou et al. 2011). To probe the relative contributions of zeamine and zeamine II in pathogenicity, we determined the virulence of the $z m s K$ mutants using the seed of rice 'Texian 25'. The results showed that the germination rates of rice seed treated with deletion mutants of $z m s A$ and $z m s K$ were approximately 91 and $79 \%$, respectively, in comparison with the untreated control, whereas expression of the wild-type zmsK restored the virulence of the mutant $\Delta \mathrm{zmsK}$ to a level similar to the wild-type strain EC1 (Fig. 6A). We noted that some infected seed grew poorly or even died post germination. Therefore, the lengths of the roots and shoots of the germinated seed were measured 7 days after inoculation. Consistent with the germination data, deletion of $z m s K$ substantially increased the growth of both shoots and leaves (Fig. 6B and C). Compared with the deletion mutants of $z m s A$, which does not produce zeamines and hardly inhibited the growth of shoots and roots, the shoots and roots of rice seed soaked with the deletion mutant of $z m s K$ grew shorter but significantly longer than the seed inoculated with the wild-type strain EC1 (Fig. 6B and C).
Complementation of the mutant $\Delta$ zmsK with wild-type $z m s K$ restored the mutant virulence but to a level slightly inferior to the wild-type EC1 (Fig. 6), which is most likely caused by the instability of the plasmid vector carrying $z m s K$ under in planta conditions. Altogether, the above results suggest that in vivo production of both zeamine and zeamine II are required for the full virulence of $D$. zeae.

\section{DISCUSSION}

Zeamine and zeamine II are the main virulence factors of the bacterial pathogen $D$. zeae, which inhibits rice seed germination and growth (Zhou et al. 2011). In addition, both zeamines are potent antibiotics (Wu et al. 2010; Zhou et al. 2012). In this study, we showed that mutation of $z m s K$, which encodes a stand-alone condensation domain NRPS (Fig. 2A), resulted in decreased antimicrobial activity and attenuated bacterial virulence (Figs. 1 and 6). Condensation domain is a conserved vital member of NRPS, and a range of different subtypes of condensation domains are known to play a key role in the formation of the peptide bond (Rausch et al. 2007). HPLC and MS analyses revealed that, unlike the control D. zeae wildtype strain EC1, which produces zeamine and zeamine II as major antibiotics and phytotoxins (Zhou et al. 2011), the $z m s K$-null mutant produced only zeamine II (Figs. 3 and 4). Notably, zeamine contains only one amide bond which links the zeamine II moiety with the octanoate group (Zhou et al. 2011). Although the overall homologies of $\mathrm{ZmsK}$ with the characterized condensation enzymes are low, ZmsK contains a short sequence "PHxxxDG" in the center of the condensation domain that is similar to the conserved "HHxxxDG" catalytic motif of condensation enzymes (Fig. 5A). Site-directed muta-
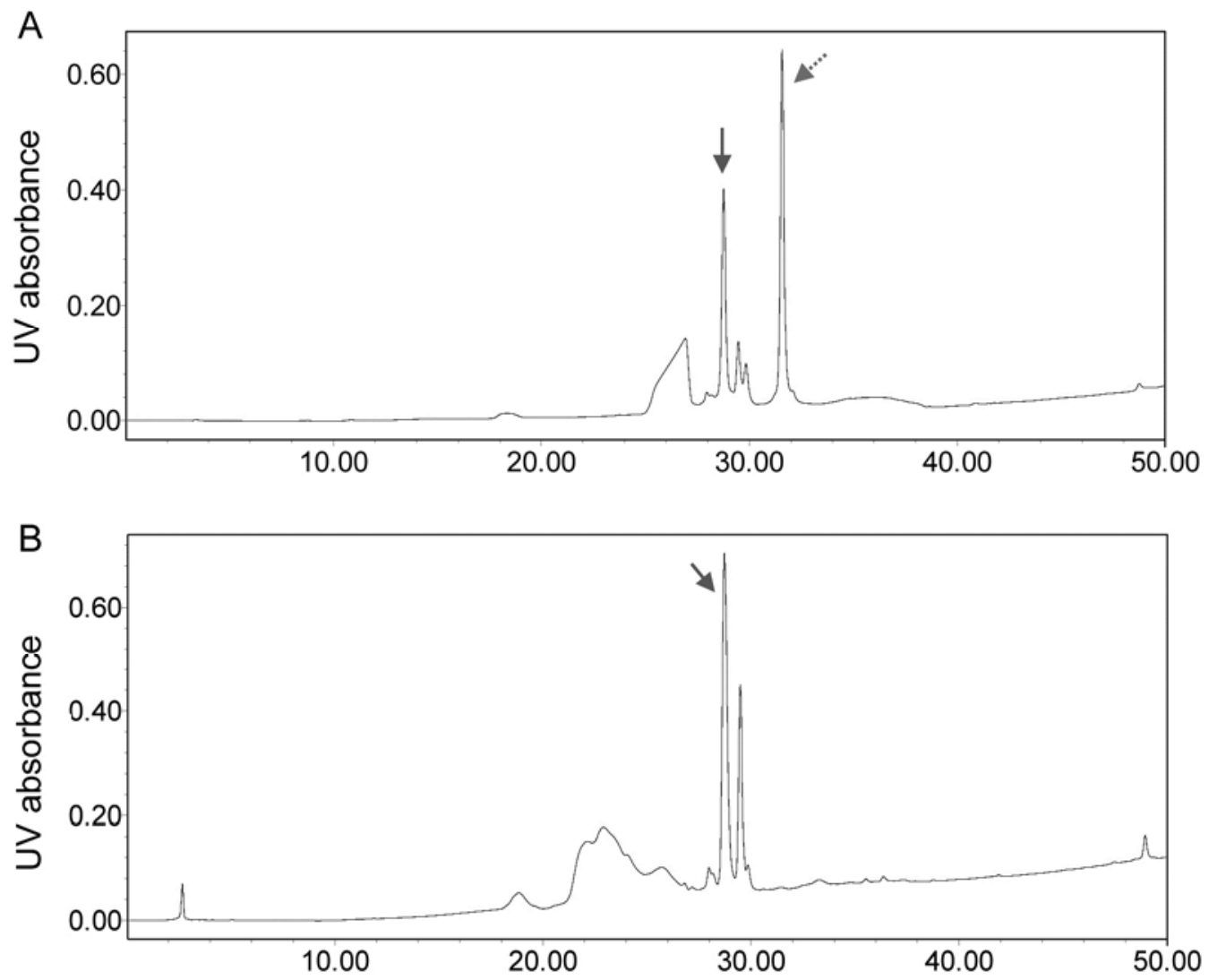

Fig. 3. High-performance liquid chromatography (HPLC) analysis of zeamine production in wild-type EC1 and the deletion mutant of $z m s K$. A, HPLC profile of EC1 extracts. B, HPLC profile of $\triangle$ zmsK extracts. Experiments were repeated at least twice with similar results. Peaks at 29.3 and 30.8 min showing strong antimicrobial activity are indicated by solid and dashed arrows, respectively. 
genesis confirmed the critical role of the "PHxxxDG" motif in the enzymatic activity of ZmsK (Fig. 5B). Cumulatively, the above findings suggest that $\mathrm{ZmsK}$ is a condensation enzyme that catalyzes the condensation reaction using zeamine II as
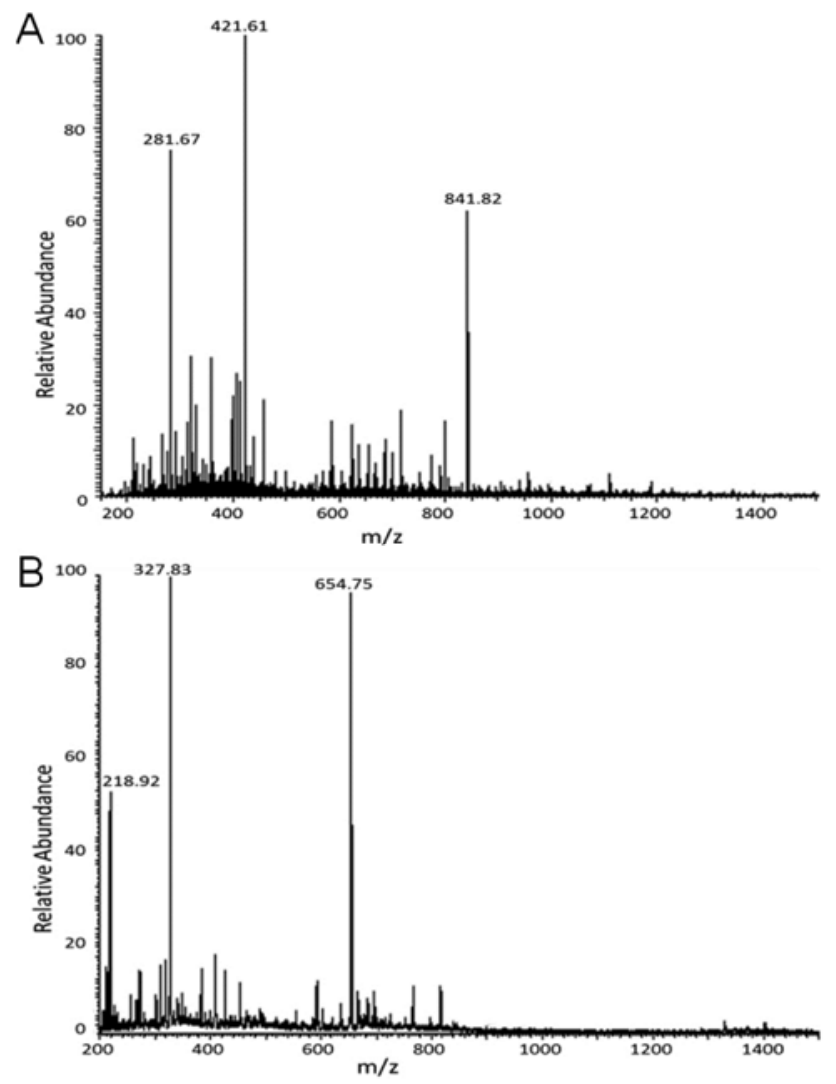

Fig. 4. Mass spectrometry (MS) analysis of zeamines from wild-type strain EC1 and the deletion mutant $\Delta \mathrm{zmsK}$. A, MS spectrum of the antimicrobial fraction at $29.3 \mathrm{~min}$. The same fractions from both EC1 and $\Delta$ zmsK showed similar MS patterns. B, MS spectrum of the antimicrobial fraction at $30.8 \mathrm{~min}$, which can be seen in the wild-type strain EC1 only. a substrate, and the other substrate is likely the thioester of octanoate (Fig. 7).

A complete NRPS enzyme typically contains various domains which are responsible for adenylation, thiolation, condensation, and termination reactions, respectively (Lautru and Challis 2004; Sieber and Marahiel 2005). ZmsK is one of the three free-standing condensation enzymes characterized so far. The other two include VibH from Vibrio cholerae and SgcC5 from Streptomyces globisporus. These two enzymes, sharing little peptide sequence homology $(<20 \%)$ with $\mathrm{ZmsK}$, are associated with the synthesis of the siderophore vibribactin and antibiotic C-1027, respectively (Keating et al. 2000; Lin et al. 2009). $\mathrm{VibH}$ is an amide synthase that carries out a condensation reaction using 2,3-dihydroxybenzoate and norspermidine as substrates in synthesis of an intermediate of vibriobactin (Keating et al. 2000), whereas SgcC5 catalyzes both ester and amide bond formation in C-1027 biosynthesis (Lin et al. 2009). Given that zeamine contains only an amide bond but not ester bond, $\mathrm{ZmsK}$ seems unlikely to be involved in formation of an ester bond in the biosynthesis of zeamine. Moreover, the homology level between $\mathrm{VibH}$ and $\mathrm{ZmsK}$ is lower than that of other homologies which contain multidomains (Supplementary Fig. S3).

While in preparation of our manuscript, a paper was published that described a gene cluster (zmn) encoding the biosynthesis of three zeamine-related antibiotics from Serratia plymuthica RVH1 (Masschelein et al. 2013). Although the sequences of RVH1 genome are not available, it was indicated that the $z m n$ gene cluster shares over $98 \%$ homology with the published gene sequences from $S$. plymuthica strain AS12 (Masschelein et al. 2013), which allows us to make comparison and bioinformatics analysis. We noted that the zmn19 gene of S. plymuthica (NCBI accession number AEF52319.1) encodes a freestanding condensation enzyme sharing approximately $48 \%$ amino acid identity with ZmsK. Similarly, we found that the polyketide synthase ZmsA from $D$. zeae shares approximately the 56\% identity with Zmn10 from S. plymuthica (NCBI accession number AEF52328.1). Zmn19 was proposed to play a role in catalysis of chain-terminating amide bond formation (Masschelein et al. 2013), which is highly consistent with our experimental findings. Because D. zeae and S. plymuthica be-

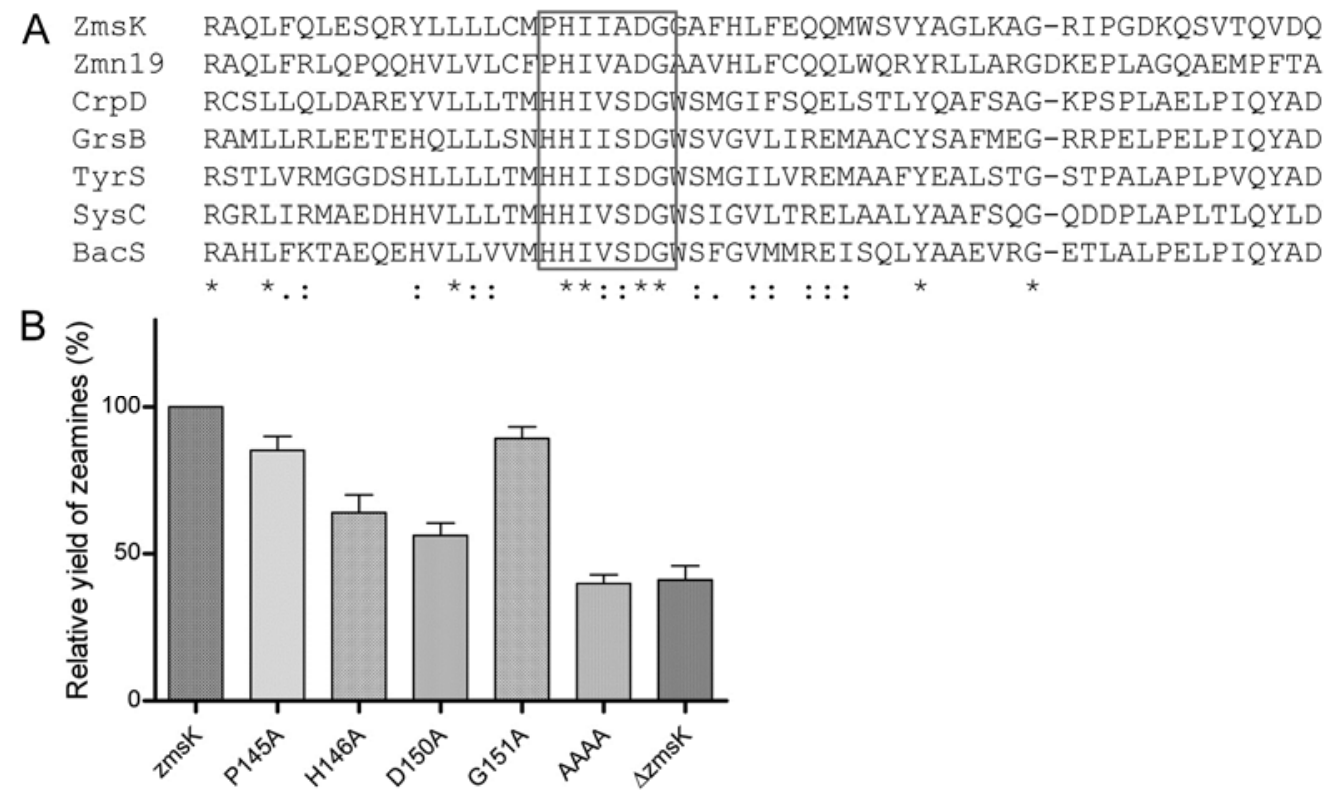

Fig. 5. Characterization of the putative catalytic motif "PHxxxDG" of ZmsK by site-directed mutagenesis. A, Partial sequence alignment of ZmsK and homologues with the conserved "HHxxxDG" motif being boxed. The condensation domain full-length sequence alignment is provided in Supplementary Fig. S2. B, Relative antimicrobial activities of ZmsK and derivatives. AAAA is the ZmsK derivative containing four substitutions (i.e., P145A, H146A, D150A, and G151A). The experiment was repeated three times with triplicates. Data shown are the means of three repeats, with error bars showing standard deviations. 
long to the $\gamma$ enterobacterials group, the relatively low sequence homology between the enzymes associated with zeamine biosynthesis in different bacterial species seems to suggest that the zeamine biosynthesis gene cluster has been acquired by corresponding bacterial pathogens long ago in the history of bacterial evolution.
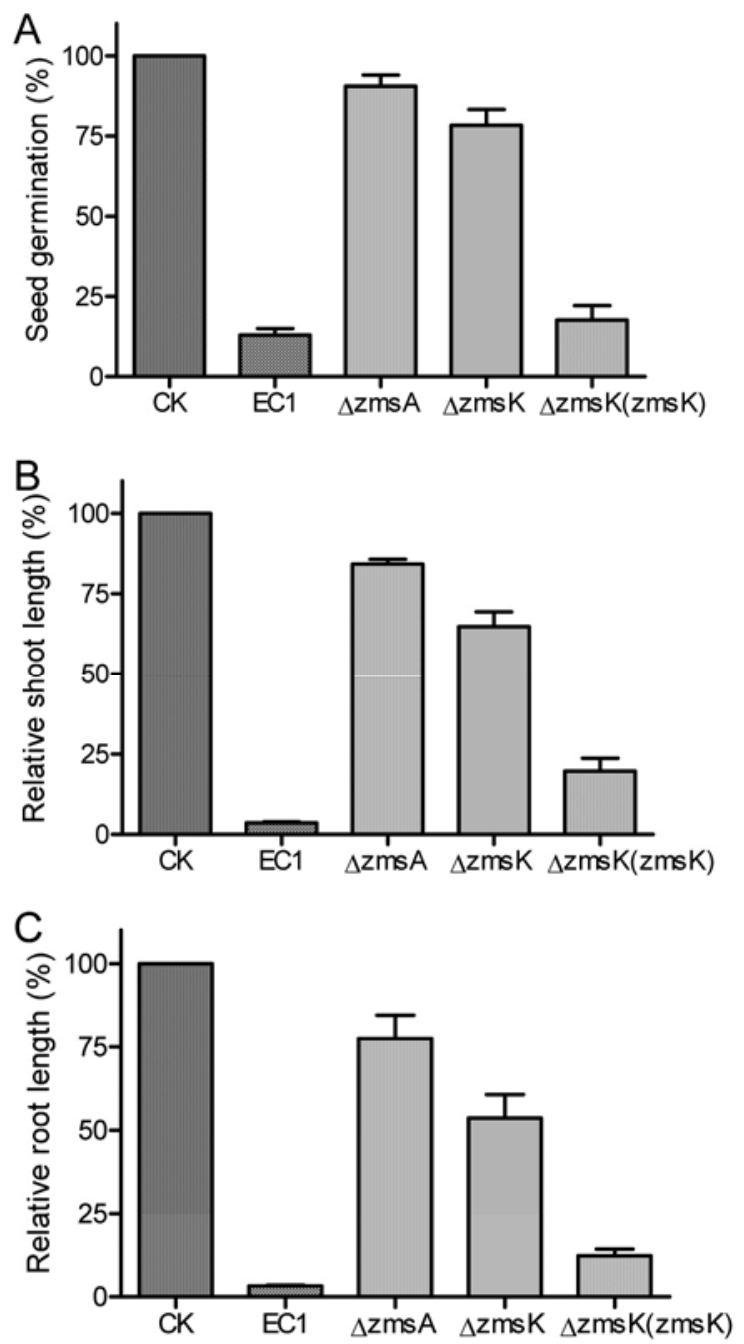

Fig. 6. Deletion of $z m s K$ attenuated bacterial virulence. A, Relative rice seed germination rate of Dickeya zeae wild-type strain EC1 and derivatives. B, Comparison of rice shoots length 7 days after inoculation. C, Comparison of rice root length 7 days after inoculation. The zeamine-null mutant $\Delta$ zmsA was used as a control. The experiment was repeated twice with 30 seeds each time. Data shown are means of 30 seeds, with error bars indicating standard deviations.
The condensation domain typically contains a conserved "HHxxxDG" motif, which is part of the active site of catalytic importance (Roongsawang et al. 2005; Stachelhaus et al. 1998). Site-directed mutagenesis analysis showed that the first histidine residue of the motif may or may not be involved in enzymatic reactions (Kraas et al. 2010; Samel et al. 2007; Stoveken et al. 2009), whereas substitution of the second histidine residue abolishes catalytic activity in most of the experiments (Imker et al. 2010; Kraas et al. 2010; Samel et al. 2007; Sieber and Marahiel, 2005; Stoveken et al. 2009). It was proposed that the second histidine in the "HHxxxDG" motif may help to promote the elongation of peptide bond (Marahiel 2009). Notably, when both histidine residues play key roles in catalysis, "D" residue of the motif may exert only little effect (Stoveken et al. 2009). However, there are also cases in which "D" residue is critical for catalytic activity while the second "H" residue generated only a little effect (Keating et al. 2002; Niu et al. 1990). There are also cases where both the second "H" and "D" of the "HHxxxDG" motif are of catalytic importance, such as the vinorine synthase (Bayer et al. 2004). Because "G" lacks a side chain, as expected, substitution of the "G" residue in the "HHxxxDG" motif seems to have only a minor or little role in catalytic reaction (Keating et al. 2002; Stoveken et al. 2009). In this study, we found a partially conserved motif " ${ }^{145} \mathrm{PHxxxDG}^{151}$ " at the center of $\mathrm{ZmsK}$, which is 296 aa in length. Site-directed mutagenesis confirmed the critical role of this motif in catalysis (Fig. 5). Similar to most previous findings, mutation of "P" and " $G$ " caused a relatively minor effect on enzymatic reaction (Fig. 5) but substitution of the " $H$ " and " $D$ " in the motif with alanine resulted in substantial reduction of catalytic activity, and mutation of "P", " $\mathrm{H}$ ", "D", and "G" simultaneously abolished the enzyme activity (Fig. 5).

Our previous study showed that zeamine is the major phytotoxin and antibiotic under in vitro culture conditions (Zhou et al. 2011). By generating the $z m s K$ mutant, which produces only zeamine II but not zeamine (Fig. 3), we were able to evaluate the relative roles of zeamine and zeamine II in pathogenesis by comparison with the $D$. zeae wild-type strain EC1 and the phytotoxin-minus mutant $\Delta$ zmsA. The results showed that mutation of $z m s K$ substantially attenuated the virulence of $D$. zeae compared with its wild-type strain but to an extent less than $\Delta$ zmsA that does not produce any zeamines, suggesting that, in planta, the bacterial pathogen could also produce zeamine as a major phytotoxin and that both zeamine and zeamine II are required for the full virulence of $D$. zeae.

In summary, the findings from this study identified a novel NRPS containing a stand-alone condensation domain, designated as ZmsK, essential for biosynthesis of the phytotoxin and antibiotic zeamine. Our results also suggest that ZmsK catalyzes the amide bond formation by using zeamine II as a substrate in

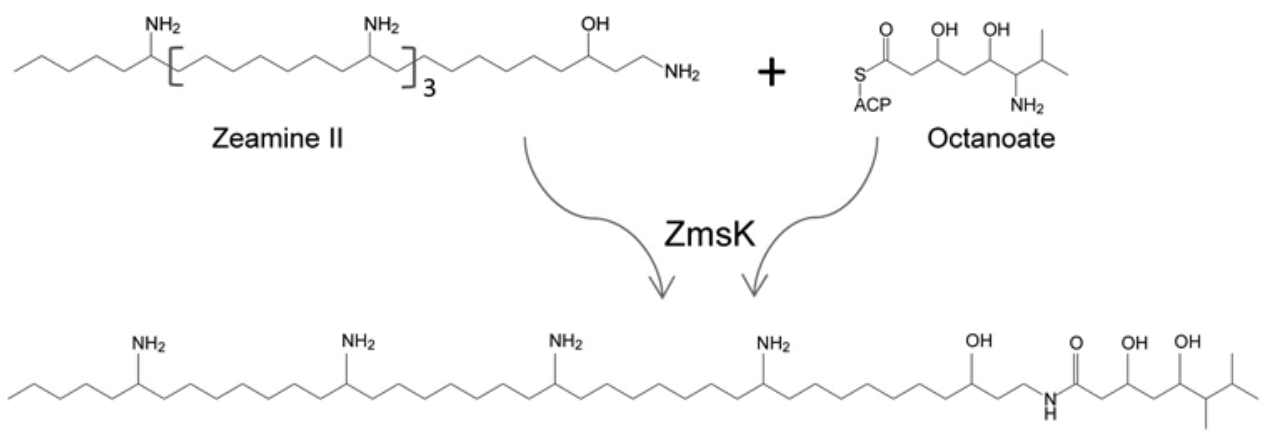

Zeamine

Fig. 7. Deduced biosynthetic route catalyzed by ZmsK. 
zeamine biosynthesis. These original findings provide groundwork for further characterization and final elucidation of the biosynthesis pathway and catalytic mechanisms of zeamines, and shed a new light on the virulence mechanisms of the bacterial pathogen $D$. zeae. Given that zeamine is a new type of antibiotic and has strong antimicrobial activities against many gram-positive and gram-negative bacterial pathogens (Wu et al. 2010), understanding its biosynthesis pathways and catalytic mechanisms may facilitate rational design of zeamine derivatives and practical application of these potent antibiotics.

\section{MATERIALS AND METHODS}

Bacterial strains and growth conditions.

The strains used for this study were listed in Table 1. D. zeae strain EC1 and its derived strains were cultivated at $28^{\circ} \mathrm{C}$ in minimal medium broth (MM), which contains $10.5 \mathrm{~g}$ of $\mathrm{K}_{2} \mathrm{HPO}_{4}, 4.5 \mathrm{~g}$ of $\mathrm{KH}_{2} \mathrm{PO}_{4}, 2 \mathrm{~g}$ of $\left(\mathrm{NH}_{4}\right)_{2} \mathrm{SO}_{4}, 2 \mathrm{~g}$ of mannitol, $2 \mathrm{~g}$ of glycerol, $0.2 \mathrm{~g}$ of $\mathrm{MgSO}_{4} \cdot 7 \mathrm{H}_{2} \mathrm{O}, 0.01 \mathrm{~g}$ of $\mathrm{CaCl}_{2}, 0.005 \mathrm{~g}$ of $\mathrm{FeSO}_{4}$, and $0.002 \mathrm{~g}$ of $\mathrm{MnCl}_{2}(\mathrm{pH}$ 7.0) per liter, with shaking at $100 \mathrm{rpm}$. E. coli strains were grown in Luria-Bertani (LB) medium, which contains $10 \mathrm{~g}$ of tryptone, $10 \mathrm{~g}$ of $\mathrm{NaCl}$, and $5 \mathrm{~g}$ of yeast extract ( $\mathrm{pH} 7.0$ ) per liter, at $37^{\circ} \mathrm{C}$ with shaking at 200 rpm. Antibiotics were added at the following concentrations when required: ampicillin, $100 \mu \mathrm{g} / \mathrm{ml}$; rifampicin, $50 \mu \mathrm{g} / \mathrm{ml}$; kanamycin, $50 \mu \mathrm{g} / \mathrm{ml}$; and streptomycin, $40 \mu \mathrm{g} / \mathrm{ml}$.

\section{DNA manipulation and preparation of expression constructs.}

The oligonucleotide primers used in this study are listed in Supplementary Table S2. Cloning of the DNA fragment containing a Tn5 insert and subsequent DNA sequencing were performed according to the methods described previously (Hussain et al. 2008; Larsen et al. 2002). For complementation of $z m s K$, the coding region of $z m s K$ was amplified by primers C18-HindIII and C18-BamHI, with the added restriction enzyme sites shown in lowercase. The PCR products were digested with BamHI and HindIII and then cloned under the control of lac $Z$ promoter in the expression vector pBBR1MCS4 digested by the same enzymes. The complementation vector was transferred into $\mathrm{DH} 5 \alpha$ and detected with primers MCS-F and MCS-R, which were designed based on the DNA sequences flanking the cloning site of pBBR1-MCS4, and conformed by DNA sequencing.

The in-frame deletion was performed by mating the wildtype strain with suicide plasmid pKNG101, which contains the fused flanking regions of the deleted fragment, and removing the plasmid after recombination, as described previously (Kaniga et al. 1991). The internal DNA fragment from 410 to
$1,117 \mathrm{bp}$ of $z m s K$ was deleted in this way. The left and right flanking fragments were amplified by using the primer pairs ORF18-1/ORF18-2 and ORF18-3/ORF18-4, respectively. Subsequently, the fusion fragment was amplified by using ORF18-1 and ORF18-4 with two flanking fragments as templates. The PCR products were digested with BamHI and SpeI and then ligated to the vector pKNG101 digested by the same enzymes to generate the construct pKNG101-zmsK.

For preparation of constructs expressing $z m s K$ derivatives, the conserved residues in the PHxxxDG motif were substituted separately using the QuickChange Site-Directed Mutagenesis kit following the procedures provided by the manufacturer (Stratagene, La Jolla, CA, U.S.A.). The mutated $z s m K$ derivatives were separately cloned under the control of lacZ promoter in the expression vector pBBR1-MCS4 for in trans complementation analysis as described below.

\section{Mutagenesis and complementation.}

Transposon mutagenesis and screening of toxin-deficient mutants were conducted following the methods described previously (Zhou et al. 2011). For generation of in-frame deletion mutant, the construct pKNG101-zmsK carried by E. coli CC118 was transformed into $D$. zeae wild-type strain EC1 by triparental mating, in which donor and receptor cells were mixed with the helper strain HB101(pRK2013) in a ratio of $2: 1: 1$ on LB plate and incubated at $28^{\circ} \mathrm{C}$ overnight. The bacterial mixtures were diluted and spread on MM plates supplemented with streptomycin to screen for transformants. After recombination, the transformants were spread on MM plates which contain $5 \%$ sucrose to make the plasmid suicide. The in-frame deletion mutants were confirmed by PCR analysis and DNA sequencing. For complementation, the expression construct pBBR1-MCS4-zms $K$ and its derivatives were separately introduced into the $z m s K$ deletion mutant by triparental mating as described above, and the transformants were selected on $\mathrm{MM}$ plates containing ampicillin. The complementation strains were confirmed by PCR analysis and DNA sequencing.

\section{Determination of antimicrobial activity.}

The antimicrobial activity was assayed using E. coli $\mathrm{DH} 5 \alpha$ as an indicator strain following the methods described previously (Zhou et al. 2011). For quantitative comparison, the units of antibiotic activity of various strains were calculated as described (Zhou et al. 2011) and converted into percentage by dividing the units of the wild-type strain EC1.

\section{HPLC and mass analyses of zeamine production.}

Bacterial supernatants were collected and processed according to a previously described method (Zhou et al. 2011), with

Table 1. Strains and plasmids used in this study

\begin{tabular}{|c|c|c|}
\hline Strain or plasmid & Relevant phenotypes and characteristics ${ }^{a}$ & Source or reference \\
\hline \multicolumn{3}{|l|}{ Strains } \\
\hline EC1 & Wild type of Dickeya zeae, Rif $^{\mathrm{r}}$ & Lab collection \\
\hline EM104 & Tn5 mutant derived from EC1, defective in zeamine production & This study \\
\hline$\Delta \mathrm{zmsK}$ & A deletion mutant derived from EC1, Rif $^{\mathrm{r}}$ & This study \\
\hline$\Delta \mathrm{zmsK}(z m s K)$ & $\Delta$ zmsK containing $z m s K$ coding region at the downstream of lac promoter, $\mathrm{Amp}^{\mathrm{r}}$ & This study \\
\hline$\Delta \mathrm{zmsA}$ & A $z m s A$ knockout mutant, defective in zeamine and zeamine II production & Zhou et al. 2011 \\
\hline $\mathrm{CC} 118$ & Escherichia coli strain as host for plasmid constructs derived from pKNG101 & Lab collection \\
\hline DH5 $\alpha$ & E. coli strain as host for plasmid constructs derived from pBBR1-MCS4 & Lab collection \\
\hline HB101(pRK2013) & Thr leu thi recA hsdR hsdM pro, $\mathrm{Km}^{\mathrm{r}}$ & Lab collection \\
\hline \multicolumn{3}{|l|}{ Plasmids } \\
\hline pKNG101 & Knockout vector, Str $^{r}$ & Lab collection \\
\hline pKNG-zms $K^{\prime}$ & pKNG101 carries the in-frame deleted fragment of $z m s K, \mathrm{Str}^{\mathrm{r}}$ & This study \\
\hline pBBR1-MCS4 & Expression vector contains a lac Z promoter, $\mathrm{Amp}^{\mathrm{r}}$ & Lab collection \\
\hline pBBR1-zms $K$ & pBBR1-MCS4 carries the coding region of $z m s K$ at down-stream of lac promoter, Amp ${ }^{\mathrm{r}}$ & This study \\
\hline pRL27 & A plasmid vector carrying $\mathrm{Tn} 5, \mathrm{Km}^{\mathrm{r}}$ & Lab collection \\
\hline
\end{tabular}

${ }^{\mathrm{a}} \mathrm{Rif}^{\mathrm{r}}, \mathrm{Amp}^{\mathrm{r}}, \mathrm{Km}^{\mathrm{r}}$, or $\mathrm{Str}^{\mathrm{r}}=$ resistance to rifampicin, ampicillin, kanamycin, or streptomycin, respectively. 
minor modifications. The supernatants were concentrated by approximately fourfold with a rotary evaporator and extracted with an equal volume of organic solvents (n-butanol/ethyl acetate $=2: 1$ ) three times. The extracts were dried by rotary evaporation and redissolved in methanol for separation by using a Phenomenex Luna $5 \mu \mathrm{C} 18$ column $(250$ by $10 \mathrm{~mm}$ ) in a semipreparative HPLC system coupled to a Waters 600 controller, 717 plus autosampler, 2487 dual $\lambda$ absorbance detector, and fraction collector II. The active elutes were collected and further separated by a reverse-phase column (Phenomenex Luna $5 \mu \mathrm{C} 8,250$ by $4.6 \mathrm{~mm}$ ) in an analytical Waters $2690 \mathrm{HPLC}$ with elutes being monitored at $\lambda=210 \mathrm{~nm}$. A gradient solvent program $\left(\mathrm{MeOH}\right.$ in $\mathrm{H}_{2} \mathrm{O}: 5 \%$ [0 to $5 \mathrm{~min}$ ], 5 to $75 \%$ [5 to 50 $\mathrm{min}$ ], 75 to $95 \%$ [50 to $51 \mathrm{~min}$ ], $95 \%$ [51 to $55 \mathrm{~min}$ ], 95 to $5 \%$ [55 to $56 \mathrm{~min}$ ], and $5 \%$ [56 to $60 \mathrm{~min}$ ]) was used for both semipreparative and analytical HPLC analysis with elution speed at 4 and $1 \mathrm{ml} / \mathrm{min}$, respectively. Elute fractions were collected for bioassay of antimicrobial activity as described in the previous section. Active fractions were characterized by MS analysis, as described previously (Zhou et al. 2011).

\section{Virulence assay.}

For rice seed germination assay, bacterial strains were grown in MM medium until the optical density at $600 \mathrm{~nm}=$ 1.5 , which were then diluted as indicated. To each tube containing $10 \mathrm{ml}$ of bacterial dilutions, 30 seeds of Texian 25 rice were added and incubated at room temperature for $5 \mathrm{~h}$. The rice seed were then washed three times with sterilized water and transferred onto three piece of filter paper, together with $5 \mathrm{ml}$ of sterilized water in a sterilized petri dish, incubated at $28^{\circ} \mathrm{C}$ with $16 \mathrm{~h}$ of light and $8 \mathrm{~h}$ of darkness for 7 days. Rice seed were treated with MM medium as a blank control.

\section{ACKNOWLEDGMENTS}

This work was supported by the National Natural Research Foundation of China (grant number 31270170, 31201479) and the Joint Research Council, Agency of Science, Technology and Research (A*Star), Singapore (grant number 10/03/FG/06/03).

\section{LITERATURE CITED}

Bayer, A., Ma, X. Y., and Stockigt, J. 2004. Acetyltransfer in natural product biosynthesis-Functional cloning and molecular analysis of vinorine synthase. Bioorg. Med. Chem. 12:2787-2795.

Goto, M. 1979. Bacterial foot rot of rice caused by a strain of Erwinia chrysanthemi. Phytopathology 69:213-216.

Hussain, M. B. B. M., Zhang, H. B., Xu, J. L., Liu, Q., Jiang, Z., and Zhang, L. H. 2008. The acyl-homoserine lactone-type quorum-sensing system modulates cell motility and virulence of Erwinia chrysanthemi pv. zeae. J. Bacteriol. 190:1045-1053.

Imker, H. J., Krahn, D., Clerc, J., Kaiser, M., and Walsh, C. T. 2010. N-Acylation during glidobactin biosynthesis by the tridomain nonribosomal peptide synthetase module GlbF. Chem. Biol. 17:1077-1083.

Kaniga, K., Delor, I. and Cornelis, G. R. 1991. A wide-host-range suicide vector for improving reverse genetics in gram-negative bacteria: Inactivation of the blaA gene of Yersinia enterocolitica. Gene 109:137-141.

Keating, T. A., Marshall, C. G., and Walsh, C. T. 2000. Vibriobactin biosynthesis in Vibrio cholera: Vibh is an amide synthase homologous to nonribosomal peptide synthetase condensation domains. Biochemistry 39:15513-15521.

Keating, T. A., Marshall, C. G., Walsh, C. T., and Keating, A. E. 2002. The structure of $\mathrm{VibH}$ represents nonribosomal peptide synthetase condensation, cyclization and epimerization domains. Nat. Struct. Biol. 9:522-526.

Kraas, F. I., Helmetag, V., Wittmann, M., Strieker, M., and Marahiel, M. A. 2010. Functional dissection of surfactin synthetase initiation module reveals insights into the mechanism of lipoinitiation. Chem. Biol. $17: 872-880$
Larsen, R. A., Wilson, M. M. Guss, A. M., and Metcalf, W. W. 2002. Genetic analysis of pigment biosynthesis in Xanthobacter autotrophicus Py2 using a new, highly efficient transposon mutagenesis system that is functional in a wide variety of bacteria. Arch. Microbiol. 178:193-201.

Lautru, S., and Challis, G. L. 2004. Substrate recognition by nonribosomal peptide synthetase multi-enzymes. Microbiology 150:1629-1636.

Lin, S. J., Van Lanen, S. G., and Shen, B. 2009. A free-standing condensation enzyme catalyzing ester bond formation in C-1027 biosynthesis. Proc. Natl. Acad. Sci. U.S.A. 106:4183-4188.

Liu, Q. G., and Wang, Z. Z. 2004. Infection characteristics of Erwinia chrysanthemi pv. zeae on rice. J. South China Agric. Univ. 25:55-57.

Liu, Y. G., and Chen, Y. L. 2007. High-efficiency thermal asymmetric interlaced PCR for amplification of unknown flanking sequences. BioTechniques 43:649-656.

Marahiel, M. A. 2009. Working outside the protein-synthesis rules: Insights into non-ribosomal peptide synthesis. J. Pept. Sci. 15:799-807.

Masschelein, J., Mattheus, W., Gao L. J., Moons, P., Houdt, R. V., Uytterhoeven, B., Lamberigts, C., Lescrinier, E., Rozenski, J., Herdewijn, P. Aertsen, A. Michiels, C., and Lavigne, R. 2013. A PKS/NRPS/FAS hybrid gene cluster from Serratia plymuthica RVH1 encoding the biosynthesis of three broad spectrum, zeamine-related antibiotics. PloS One 8:e54143.

Nassar, A., Bertheau, Y., Dervin, C., Narcy, J. P., and Lemattre, M. 1994. Ribotyping of Erwinia chrysanthemi strains in relation to their pathogenic and geographic distribution. Appl. Environ. Microbiol. 60:37813789.

Niu, X. D., Stoops, J. K., and Reed, L. J. 1990. Overexpression and mutagenesis of the catalytic domain of dihydrolipoamide acetyltransferase from Saccharomyces cerevisiae. Biochemistry 29:8614-8619.

Rausch, C., Hoof, I., Weber, T., Wohlleben, W., and Huson, D. H. 2007. Phylogenetic analysis of condensation domains in NRPS sheds light on their functional evolution. BMC Evol. Biol. 7:78.

Roongsawang, N., Lim, S. P., Washio, K. Takano, K., Kanaya, S., and Morikawa, M. 2005. Phylogenetic analysis of condensation domains in the nonribosomal peptide synthetases. FEMS (Fed. Eur. Microbiol. Soc.) Microbiol. Lett. 252:143-151.

Samel, S. A., Schoenafinger, G., Knappe, T. A., Marahiel, M. A., and Essen, L. O. 2007. Structural and functional insights into a peptide bond-forming bidomain from a nonribosomal peptide synthetase. Structure. 15:781-792.

Samson, R., Legendre, J. B., Christen, R., Fischer-Le, Saux M., Achouak, W., and Gardan, L. 2005. Transfer of Pectobacterium chrysanthemi (Burkholder et al. 1953) Brenner et al. 1973 and Brenneria paradisiaca to the genus Dickeya gen. nov. as Dickeya chrysanthemi comb. nov. and Dickeya paradisiaca comb. nov. and delineation of four novel species, Dickeya dadantii sp. nov., Dickeya dianthicola sp. nov., Dickeya dieffenbachiae sp. nov. and Dickeya zeae sp. nov. Int. J. Syst. Evol. Microbiol. 55:1415-1427.

Sieber, S. A., and Marahiel, M. A. 2005. Molecular mechanisms underlying nonribosomal peptide synthesis: Approaches to new antibiotics. Chem. Rev. 105:715-738.

Sinha, S. K., and Prasad, M. 1977. Bacterial stalk rot of maize, its symptoms and host-range. Zentralbl. Bakteriol. Parasitenkd. Infektkrankh. Hyg. Abt. 1 Orig. Reihe A 132:81-88.

Stachelhaus, T., Mootz, H. D., Bergendahl, V., and Marahiel, M. 1998. Peptide bond formation in nonribosomal peptide biosynthesis. Catalytic role of the condensation domain. J. Biol. Chem. 273:22773-22781.

Stead, D. E., Parkinson, N., Bew, J., Hennessy, J., Wilson, J. K., and Elphinstone, J. G. 2010. The first record of Dickeya zeae in the UK. Plant Pathol. 59:401-401.

Stoveken, T., Kalscheuer, R., and Steinbuchel, A. 2009. Both histidine residues of the conserved HHXXXDG motif are essential for wax ester synthase/acyl-CoA:diacylglycerol acyltransferase catalysis. Eur. J Lipid Sci. Technol. 111:112-119.

Wu, J., Zhang, H. B., Xu, J. L. Cox, R. J., Simpson, T. J., and Zhang, L. H. 2010. (13)C Labeling reveals multiple amination reactions in the biosynthesis of a novel polyketide polyamine antibiotic zeamine from Dickeya zeae. Chem. Commun. 46:333-335.

Zhou, J., Zhang, H. B., Wu, J., Liu, Q., Xi, P., Lee, J., Liao, J., Jiang, Z., and Zhang, L. H. 2011. A novel multidomain polyketide synthase is essential for zeamine production and the virulence of Dickeya zeae. Mol. Plant-Microbe Interact. 24:1156-1164.

\section{AUTHOR-RECOMMENDED INTERNET RESOURCE}

EMBL SMART database: smart.embl-heidelberg.de 